\title{
RECURSIVELY SATURATED MODELS OF SET THEORY
}

\author{
JOHN STEWART SCHLIPF
}

\begin{abstract}
We determine when a model $\mathfrak{M}$ of $\mathrm{ZF}$ can be expanded to a model $\langle\mathfrak{M}, \mathfrak{X}\rangle$ of a weak extension of Gödel Bernays: $\mathrm{GB}+$ the $\Delta_{1}^{1}$ comprehension axiom. For nonstandard $\mathfrak{M}$, the ordinal of the standard part of $\mathfrak{M}$ must equal the inductive closure ordinal of $\mathfrak{M}$, and $\mathfrak{M}$ must satisfy the axioms of $Z$ F with replacement and separation for formulas involving predicates for all hyperelementary relations on $\mathfrak{M}$. We also consider expansions to models of $G B+\Sigma_{1}^{1}$ choice, observe that the results actually apply to more general theories of well-founded relations, and observe relationships to expansibility to models of other second order theories.
\end{abstract}

In this paper we consider when a model $\mathfrak{M}$ of $\mathrm{ZF}$ (Zermelo-Fraenkel set theory) can be expanded to a model $\langle\mathfrak{M}, \mathfrak{X}\rangle$ of a weak set-and-class theory-stronger than GB (Gödel-Bernays) but much weaker than MK (Morse-Kelley). Thus $\mathfrak{X}$ will be a collection of classes-a collection of subsets of $\mathfrak{M}$.

We shall consider $\langle\mathfrak{M}, \mathfrak{X}\rangle$ to be a Henkin model for second order logic. Thus second order (capital letter) variables vary over classes-elements of $\mathfrak{X}$-and first order (small letter) ones, over sets-elements of $\mathfrak{M}$. A formula is said to be first order if it contains no class quantifiers-it may have class parameters. So a $\Sigma_{1}^{1}$ formula is a formula of the form $\exists X \varphi(X, Y, z)$, where $\varphi$ is first order. (We actually wish to allow a sequence of class parameters $Y$ and a sequence of set parameters $z$, but we include only one of each for simplicity of writing. We follow this convention henceforth.) A $\Pi_{1}^{1}$ formula is one of the form $\forall X \varphi(X, Y, z)$ for first order $\varphi$. We say $Y \subseteq \mathfrak{M}$ is $\Delta_{1}^{1}$ definable if it is definable in $\langle\mathfrak{M}, \mathfrak{X}\rangle$ by both $\Sigma_{1}^{1}$ and $\Pi_{1}^{1}$ formulas. We say $\langle\mathfrak{M}, \mathfrak{X}\rangle \vDash \Delta_{1}^{1} \mathrm{CA}\left(\Delta_{1}^{1}\right.$ comprehension axiom) if every $\Delta_{1}^{1}$ definable subset of $\mathfrak{M}$ is an element of $\mathcal{X}$. (This can be expressed with an obvious axiom scheme.) We ask when $\mathfrak{M} \vDash \mathrm{ZF}$ can be expanded to a model $\langle\mathfrak{M}, \mathfrak{X}\rangle \vDash \mathrm{GB}+\Delta_{1}^{1}$ CA. That the problem is not trivial is suggested by the following two well-known results: If $\mathfrak{M} \vDash Z F$ and $\mathscr{X}$ is the collection of definable subsets of $\mathfrak{M}$, then $\langle\mathfrak{M}, \mathfrak{X}\rangle \vDash \mathrm{GB}$. And if $\langle\mathfrak{M}, \mathfrak{X}\rangle \vDash M K, \mathfrak{M} \vDash \operatorname{Con}(Z F)$. (For this we need only that

Received by the editors March 31, 1978 and, in revised form, July 16, 1979; principal results were presented at the annual meeting of the Association for Symbolic Logic in St. Louis, Missouri, January 28, 1977.

AMS (MOS) subject classifications (1970). Primary 02B15, 02F27, 02H20, 02K99; Secondary 02B25, $02 \mathrm{H} 10,02 \mathrm{~K} 20$.

Key words and phrases. Model of set theory, nonstandard model of set theory, recursively saturated model, inductive closure ordinal, hyperelementary relation, next admissible set, well-founded parts of models, $\Delta_{1}^{1}$ comprehension, $\Sigma_{1}^{1}$ choice.

${ }^{1}$ The preparation of this paper was partially supported by NSF Grant MCS-76-17254. 
$\langle\mathfrak{M}, \mathfrak{X}\rangle \vDash \mathrm{GB}+\Delta_{1}^{1} \mathrm{CA}+\mathrm{a} \Pi_{1}^{1}$ foundation scheme.) Moschovakis, in [1971], discusses a theory that looks very similar to ours-GB $+\Delta_{1}^{1} \mathrm{CA}+$ schemes asserting that every $\Pi_{1}^{1}$ or $\Sigma_{1}^{1}$ definable class has a minimal element-and proves noticeably different results.

This paper generalizes results in Barwise and Schlipf [1976] to models of ZF. The techniques in this paper, however, can easily be applied to that paper to yield two improvements: first, we deal with $\Delta_{1}^{1} \mathrm{CA}$ instead of the more complicated, and apparently stronger, essential $\Delta_{1}^{1} \mathrm{CA}$. And second, in noting the connection with Theorems 6, 7 and 8, we observe that $\mathrm{GB}+\Delta_{1}^{1} \mathrm{CA}$ is in some sense a maximal "conservative extension for sentences about sets" of ZF (thus giving a mathematical justification for looking at that theory). The same proofs give essentially the same results for Peano arithmetic plus the foundation axiom $\forall X \exists y$ ( $y$ is the least element of $X$ or $X=0$ ).

In fact, the methods are mostly quite general and can be applied to any structure $\mathfrak{M}$ with a partial ordering < (which we wish to make look like a well-founded relation) and an inductive pairing function (apparently a technical necessity) to see whether there is a nonempty set $\mathcal{X}$ of subsets of $\mathfrak{M}$ so that $\langle\mathfrak{M}, \mathfrak{X}\rangle \vDash \Delta_{1}^{1}$ $\mathrm{CA}+\forall X \exists y(y$ is a $<-$ minimal element of $X$ or $X \cap$ field $(<)=0)$. (If the pairing function is not definable, but still inductive, we may have to take $\mathcal{X} \subseteq \mathfrak{D}^{k}$ for some integer $k$.) Adding the GB axioms only complicates matters: the foundation axiom above is provable in GB, but there we must worry about the comprehension and replacement axioms. The reader can easily modify the results for either of the above two cases. Peano arithmetic is noticeably simpler in that all nonstandard models are non- $\omega$-standard.

Some similar theorems were proved roughly at the same time by Bielinski, who, in [1977], published results analogous to Theorems 3, 4, 5, 7, and 8. He followed the paper (Barwise and Schlipf [1976]) closely and thus missed the two improvements of this paper over the Barwise-Schlipf approach noted above. (Accordingly, he was able to use the Moschovakis result cited as a weaker form of Theorem 1 [1974, Theorem 7F.1] plus the Barwise-Schlipf proof rather than proving Theorem 1 itself.) He does not seem to have observed, as we did in the paragraph above, that Theorems 3 through 8 actually hold in great generality, whereas here we concentrate upon the set theory case-which seems the most interesting example after Peano arithmetic-to simplify the paper.

Our basic tools are Theorems 1 and 2, below.

THEOREM 1 [KLEENE FOR THE INTEGERS; MOSCHOVAKIS FOR ACCEPTABLE STRUCTURES; HARRINGTON-KIROUSIS-SCHLIPF IN THIS GENERALITY]. Let $\mathfrak{M}$ be a structure with a definable pairing function. Then the smallest nonempty set $\mathcal{X}$ of subsets of $\mathfrak{M}$ such that $\langle\mathfrak{M}, \mathfrak{X}\rangle \vDash \Delta_{1}^{1} C A$ is the set of hyperelementary subsets of $\mathfrak{M}$. (See Harrington, Kirousis and Schlipf [1978] for the proof.)

Definition. Let $\kappa^{\mathfrak{M}}$ denote the inductive closure ordinal of $\mathfrak{M}$.

One half of Theorem 1 -that the hyperelementary subsets of $\mathfrak{M}$ really do give a model of $\Delta_{1}^{1} \mathrm{CA}$-follows from the generalized Barwise-Gandy-Moschovakis result 
that, over any structure $\mathfrak{M}$ with an inductive pairing function, the set of hyperelementary subsets of $\mathfrak{M}$ is just the set of subsets of $\mathfrak{M}$ in the next admissible set-and thus the result follows by $\Delta_{1}$ comprehension in admissible sets. By the same results $\kappa^{\mathfrak{R}}$ is just the ordinal of the admissible set. (See Barwise, [1975, Chapter VI], for the general results.) (This is the only place in the general treatment that we need to know that $\mathfrak{M}$ has an inductive pairing function.)

Definition. $L(\alpha)$ is the set of sets constructible by ordinal $\alpha$. For $A$ a transitive set, $\mathcal{L}_{A}$ is the infinitary language $\mathcal{L}_{\infty \omega} \cap A$.

We shall also use the following result:

THEOREM 2. Let $\mathfrak{M}$ be a structure with an inductive pairing function. The set of hyperelementary subsets of $\mathfrak{M}$ is just the set of subsets of $\mathfrak{M}$ definable in the language $\mathcal{L}_{L\left(\kappa^{\mathfrak{P}}\right)}$ (with parameters from $\mathfrak{M}$ ). (This follows from Corollary II.5.6 of Schlipf [1977].)

Definition. Let $\mathcal{H} \mathcal{E}_{\mathfrak{M}}$ denote the set of hyperelementary subsets of $\mathfrak{M}$.

Definition. For $A$ a transitive set, let $\mathrm{ZF}_{A}$ denote the $\mathrm{ZF}$ axioms with replacement and separation axioms for all formulas in $\mathcal{L}_{A}$.

THEOREM 3. Let $\mathfrak{M} \vDash Z F$. The following are equivalent:

(i) $\exists \mathfrak{X}\langle\mathfrak{M}, \mathfrak{X}\rangle \vDash G B+\Delta_{1}^{1} C A$,

(ii) $\left\langle\mathfrak{M}, \mathcal{H} \varepsilon_{\mathfrak{R}}\right\rangle \vDash G B+\Delta_{1}^{1} C A$,

(iii) $\mathfrak{M} \vDash Z F_{L\left(\kappa^{\mathfrak{P}}\right)}$.

Proof. (ii) $\Rightarrow$ (i) vacuously, and that (i) $\Rightarrow$ (ii) is easy to check, for $\Delta_{1}^{1}$ CA always holds in $\mathcal{H} \mathcal{E}_{\mathfrak{M}}$, and the class formation axioms of GB follow from that, while the rest of the axioms of GB follow easily from $\mathcal{H} \mathcal{E}_{\mathfrak{M}}$ 's being a subset of any such $\mathcal{X}$. That (ii) $\Rightarrow$ (iii) is immediate from Theorems 1 and 2 -it is just the separation and replacement axioms of GB. For (iii) $\Rightarrow$ (ii) we need only check the GB part, by Theorem 1, and that is just what (iii) asserts.

We get extra information about the non-well-founded examples.

THEOREM 4. Let $\mathfrak{M} \vDash Z F$ be non-well-founded; $\beta=$ ord(well-founded part of $\mathfrak{M})$. Suppose $\exists \mathfrak{X}\langle\mathfrak{M}, \mathfrak{X}\rangle \vDash G B+\Delta_{1}^{1} C A$. Then $\beta=\kappa^{\mathfrak{M}}$.

Proof. Clearly $\beta \leqslant \kappa^{\mathfrak{M}}$. But if $\beta<\kappa^{\mathfrak{M}}$, then the well-founded part of $\mathfrak{M}$ is hyperelementary, and its complement has no minimal element, violating foundation.

It is an obvious guess that if $\mathfrak{M} \vDash Z F$ and the ordinal of the well-founded part of $\mathfrak{M}$ is $\kappa^{\mathfrak{M}}$, then $\mathfrak{M}$ can be expanded to a model of $\mathrm{GB}+\Delta_{1}^{1} \mathrm{CA}$. But this is false-at least under a suitable nontriviality condition, such as there existing a standard model of ZF. The guess is obviously true if $\kappa^{\mathfrak{M}}=\omega$ (by Theorem 3). But also by Theorem 3 , if $\mathfrak{M}$ is so expandable and $\kappa^{\mathfrak{M}}>\omega$, then $\mathfrak{M} \vDash \exists \alpha\left(R(\alpha)<\mathfrak{E}_{\omega \omega} \mathfrak{M}\right)$ (which is a perfectly good sentence of $\mathcal{E}_{L\left(\omega_{1}^{c k}\right)}$ ). This fails in the minimal model of ZF. By Barwise compactness, it also fails in a model the ordinal of whose well-founded part is $\omega_{1}^{c k}$, and, also by Barwise compactness, we can choose such an $\mathfrak{M}$ with $\kappa^{\mathfrak{M}}=\omega_{1}^{c k}$. (See Ressayre [1977, Proposition II.1] or Schlipf [1978, Theorem 3.3] for this last construction.) 
Definition. Let $D f_{\alpha}(\mathfrak{M})$ denote the subsets of $\mathfrak{M}$ definable by $\mathcal{L}_{L(\alpha)}$ formulas (with parameters from $\mathfrak{M}$ ).

By combining the methods of Theorems 3 and 4 we get:

TheOREM 5. Suppose $\left\langle\mathfrak{M}, D f_{\alpha}(\mathfrak{M})\right\rangle \vDash G B+\Delta_{1}^{1} C A$, $\mathfrak{M}$ non-well-founded. Then the ordinal of the well-founded part of $\mathfrak{M}=\kappa^{\mathfrak{M}}=\alpha$.

THEOREM 6. $G B+\Delta_{1}^{1} C A$ is a conservative extension of $Z F$ for sentences about sets; i.e., if $\varphi$ is a formula containing only set variables and $G B+\Delta_{1}^{1} C A \vdash \varphi$, then $Z F \vdash \varphi$. Similarly, for each countable admissible ordinal $\alpha, G B+\Delta_{1}^{1} C A+$ the assertion that for each $\beta<\alpha$, the model is $\beta$ standard, is a conservative extension of $Z F_{L(\alpha)}$

Proof. For the first part we note that every model of ZF has a recursively saturated elementary extension-thus an elementary extension $\mathfrak{D}$ where $\kappa^{\mathfrak{M}}=\omega$, and hence an elementary extension expandable to a model of $\mathrm{GB}+\Delta_{1}^{1} \mathrm{CA}$. The second part is similar.

We are now ready to consider the mathematical justification of looking at the specific theory GB $+\Delta_{1}^{1} \mathrm{CA}$. This we argue on the basis of the theory's nice model theory. We already know that $\mathrm{GB}+\Delta_{1}^{1} \mathrm{CA}$ is a conservative extension of $\mathrm{ZF}$ for sentences about sets. Theorem 6 will tell us that it also is a maximal conservative extension in the following sense: for a countable non- $\omega$-standard model $\mathfrak{M}$ of $\mathrm{ZF}$, while expandability to a model of $\mathrm{GB}+\Delta_{1}^{1} \mathrm{CA}$ has no first order $\left(\mathfrak{L}_{\omega \omega}\right)$ consequences-telling us only something about the model-theoretic structure of $\mathfrak{M}$, the additional consequences of expandability to a stronger r.e. theory $T$ are all first order. For countable, non-well-founded $\mathfrak{M}$ whose standard part has ordinal $\alpha$ we get an analogous result: the $L(\alpha)$ consequences of $\mathrm{GB}+\Delta_{1}^{1} \mathrm{CA}$ not involving class variables are just the consequences of the fairly obvious theory $\mathrm{ZF}_{L(\alpha)}$, while the additional consequences of stronger $L(\alpha)$-r.e. theories are all expressable by $L(\alpha)$ r.e. theories without class variables.

Theorem 7 is a special case of a theorem proved independently by Barwise and Ressayre. Theorem 8 is a special case of a theorem of Ressayre.

TheOREM 7 [BARWISE AND RESSAYRe, INDEPENDENTLy]. Let $\mathfrak{M}$ be countable, $\mathfrak{R} \vDash Z F$. Suppose that the next admissible set above $\mathfrak{M}$ has ordinal $\omega$ (i.e., since we just said $\mathfrak{M} \vDash Z F, \kappa^{\mathfrak{M}}=\omega$ ). Let $T$ be an r.e. theory of models $\langle\mathfrak{N}, \mathcal{Y}\rangle$ of some theory of sets and classes. Suppose $\mathfrak{M}$ satisfies all first order consequences of $T$ (not involving class variables). Then $\mathfrak{M}$ can be expanded to a model $\langle\mathfrak{M}, \mathfrak{X}\rangle \vDash T$.

(A proof can be found in Theorem II.4 of Ressayre [1977]; another can be found in Theorem II.1.1 of Schlipf [1977]. The next theorem is a special case of Theorems II.4 and III.4 of Ressayre [1977].)

ThEOREM 8 [RESSAYRE]. Let $\mathfrak{M}$ be countable, $\mathfrak{M} \vDash Z F$. Suppose the next admissible set above $\mathfrak{M}$ has ordinal $\alpha$ (i.e., in this case, that $\kappa^{\mathfrak{M}}=\alpha$ ). Let $T$ be a $\Sigma$ theory of $L(\alpha)$ of models $\langle\mathfrak{N}, \mathcal{Y}\rangle$ of some theory of sets and classes. Suppose $\mathfrak{M}$ satisfies all $L(\alpha)$ consequences (with no class variables) of $T$. Then $\mathfrak{M}$ can be expanded to a model of $T$. 
Thus, for $T$ a theory with GB $+\Delta_{1}^{1} \mathrm{CA} \subseteq T$, we can think loosely of factoring $T$ : $\mathrm{GB}+\Delta_{1}^{1} \mathrm{CA}$ is the part of $T$ that tells us about structural properties of the countable models, and the rest of the theory is the part that tells us about first order consequences.

An obvious question arises here: what is the strength of the (set theoretic) axiom asserting that there is an $\alpha$-standard model of GB $+\Delta_{1}^{1}$ CA? For the case $\alpha=\omega$ this is just equivalent to the assertion that $\mathrm{ZF}$ is consistent, for if $\mathrm{ZF}$ is consistent, it has a recursively saturated model $\mathfrak{M}$, which can be expanded to a model of $\mathrm{GB}+\Delta_{\mathrm{l}}^{1} \mathrm{CA}$ since $\kappa^{\mathfrak{M}}=\omega$. Similarly, for countable $\alpha$, the assertion that there is an $\alpha$-standard model of $\mathrm{GB}+\Delta_{1}^{1} \mathrm{CA}$ is equivalent to the assertion that the infinitary theory $\mathrm{ZF}_{L\left(\alpha^{+}\right)}$is consistent, for $\alpha^{+}$the least admissible greater than $\alpha$. The assertion that there is a well-founded model of $\mathrm{GB}+\Delta_{1}^{1} \mathrm{CA}$ is obviously much stronger. For example, if some $X,\langle L(\alpha), \mathfrak{X}\rangle \vDash \mathrm{GB}+\Delta_{1}^{1} \mathrm{CA}$, then, for $\alpha^{+}$the least admissible above $\alpha$, for all $\beta<\alpha$, all subsets of $\beta$ in $L\left(\alpha^{+}\right)$are also in $L(\alpha)$. On the other hand, the assertion that there is a well-founded model of GB $+\Delta_{1}^{1}$ CA-in fact, that there is a standard model of Morse-Kelley-is known to follow from the existence or an inaccessible cardinal or a cardinal inaccessible in $L$.

These results give special emphasis to an obvious program: to look at various theories extending $\mathrm{GB}+\Delta_{1}^{1} \mathrm{CA}$ and study their first order consequences. $\mathrm{Z}$. Ratajczyk has recently done this for GB + the $\Sigma_{n}^{1}$ class formation scheme (for each $n$ ) and thus also for Morse-Kelley. The author of this paper has also (independently) found a recursive axiomatization of the first order consequences of MorseKelley. We study here one additional axiom and both its finitary and infinitary consequences.

Definition. The axiom scheme of $\Sigma_{1}^{1}$ choice $\left(\Sigma_{1}^{1} \mathrm{AC}\right)$ is the scheme

$$
\forall x \exists X \varphi(X, x, Z, z) \Rightarrow \exists X \forall x \varphi\left(X_{x}, x, Z, z\right)
$$

for arbitrary parameters $Z$ and $z$, and $X_{x}$ the section of $X$ determined by $x$-thus $\{y:\langle x, y\rangle \in X\}$, and for $\varphi$ first order.

It is well known that $\mathrm{GB}+\Sigma_{1}^{1} \mathrm{AC} \vDash \Delta_{1}^{1} \mathrm{CA}$.

THEOREM 9 [WeLl KNOWN]. If $\langle\mathfrak{D}, \mathcal{X}\rangle \vDash G B+\Sigma_{1}^{1} A C$, then $\mathfrak{M} \vDash A C$.

THEOREM 10. If $\mathfrak{M} \vDash Z F+(V=O D)$ and $\mathfrak{M}$ can be expanded to a model of $G B+\Delta_{1}^{1} C A$, then $\left\langle\mathfrak{M}, \mathcal{H}_{\mathcal{F}_{\mathfrak{M}}}\right\rangle \vDash G B+\Sigma_{1}^{1} A C$.

Proof. An obvious $\Sigma_{1}^{1}$ collection axiom clearly holds in $\mathcal{C}_{\mathfrak{F}}$ : $\forall x \exists Y \varphi(x, Y, Z, z) \Rightarrow \exists Y \forall x \exists q \varphi\left(x, Y_{q}, Z, z\right)$; this is easily provable from $\Sigma$ collection in the next admissible set above $\mathfrak{M}$ plus some coding. Now we can use the canonical well-ordering given by $(V=O D)$ to pick the least such $q$.

Between Theorems 9 and 10 there is a large gap-the gap between the first order $\mathrm{AC}$ and $(V=O D)$. In general, this gap seems very difficult to fill. However, in the case of countable non-well-founded models we can settle the question. The result follows from the following generalization of a theorem of Cohen, Felgner, Jensen, Kripke, and Solovay (all independently). 
THEOREM 11. Let $A$ be a countable admissible set; let $\mathfrak{M}$ be a countable model of $Z F_{A}$; and suppose that for each $\beta$ less than the ordinal of $A, \beta$ is in the well-founded part of $\mathfrak{M}$ (or rather, in the transitive collapse of the well-founded part of $\mathfrak{M}$ ). Suppose $\mathfrak{M} \vDash A C$. Then there is a binary relation $\leqslant$ on $\mathfrak{M}$ such that $\langle\mathfrak{M}, \leqslant\rangle \vDash$ " $\leqslant$ is a well-ordering of $\mathfrak{M}$ " $+Z F_{A}$ with replacement and separation for formulas involving $\leqslant$.

Proof. The proof is a relatively easy modification of the standard case, that of $A=$ the set of hereditarily finite sets. See, e.g., Gaifman [1975] for a proof of the standard case.

We use model-theoretic forcing for the admissible language $\mathscr{L}_{A}$. (See, e.g., Keisler [1973].) We let $\mathcal{L}^{\prime}=\mathfrak{L}(\leqslant)$, and we add a name $\hat{m}$ for each element $m$ of $\mathfrak{M}$-these are the only names. We define our notion of forcing $\mathcal{P}$ by: $p \in \mathcal{P}$ if $p \in \mathfrak{M}$ and $\mathfrak{M} \vDash$ ( $p$ is a well-ordering of some $R(\kappa)$ ). Set $p \leqslant q$ if $p$ is an initial subsequence of $q$. (Thus $\mathcal{P}$ is closed under unions of chains.) Say $p \Vdash(\hat{x} \in \hat{y})$ iff $x \in y ; p \Vdash(\hat{x}=\hat{y})$ iff $x=y$; and $p \Vdash(\hat{x} \leqslant \hat{y})$ iff $\langle x, y\rangle \in p$.

We may assume the well-founded part of $\mathfrak{M}$ is a transitive set with the real $\in$ relation. We show $A \subset \mathfrak{M}$ by induction on the rank of elements of $A$ and then show that $\mathfrak{M}$ satisfies reflection with respect to $A$-finite sets of $\mathfrak{L}_{A}$ formulas (much as for finitary logic). Since $\mathfrak{M}$ and $\mathfrak{M}[G]$ will have the same elements, we just need to prove replacement and separation in $\mathfrak{M}[G]$.

For replacement we show that if $p \Vdash \forall x \in a \exists y \varphi(x, y)$, then $p \Vdash^{w} \exists b$ $\forall x \in a \exists y \in b \varphi(x, y)$, i.e., that $\forall p^{\prime} \geqslant p \exists r^{\prime} \geqslant p^{\prime} \quad\left(r^{\prime} \Vdash \exists b \forall x \in a \exists y \in b \varphi(x, y)\right)$. (Parameters in $\varphi$ are carried along easily.) Fix $p^{\prime} \geqslant p . p^{\prime} \vDash \forall x \in a \exists y \varphi(x, y)$, so

$$
\forall x \in a \forall q \geqslant p^{\prime} \exists r \geqslant q \exists y(r \vDash \varphi(x, y)) \text {. }
$$

By reflection in $\mathfrak{M}$, choose a cardinal $\kappa$ (in the sense of $\mathfrak{M}$ ) so that: (1) $p^{\prime}, a, \varphi$, and all parameters of $\varphi$ are in $R(\kappa)$; (2) the definitions of $\mathcal{P}$ and of $q \Vdash \varphi$ (the fixed $\varphi$ above) reflect to $R(\kappa)$; (3) $R(\kappa)$ satisfies (*); and (4) $(\operatorname{cof}(\kappa)>\operatorname{card}(a))^{(\mathfrak{R})} . \mathfrak{M} \vDash A C$, so pick an $S$ in $\mathfrak{M}$ so that $\mathfrak{M} \vDash$ " $S$ well-orders $R(\kappa)$." By recursion inside $\mathfrak{M}$ we define an increasing sequence of conditions $r_{\alpha+1}$ in $R(\kappa)$ forcing that for some $y \in R(\kappa), \varphi\left(x_{\alpha}, y\right)$, where $x_{\alpha}$ is the $\alpha$ th element of $a$ under the ordering $S \mid a$. $r_{0}=p^{\prime} . r_{\alpha+1}$ is the $S$-least condition in $R(\kappa)$ forcing $\varphi\left(x_{\alpha}, y\right)$ and extending $r_{\alpha}$. (Such a condition exists by (3).) At limit ordinals take unions. Finally, let $\boldsymbol{r}^{\prime}=$ $\cup\left\{r_{\alpha+1}: x_{\alpha} \in a\right\}$. (We are assuming $a \neq 0$.) Clearly $r^{\prime} \Vdash \forall x \in a \exists y \in R(\kappa) \varphi(x, y)$. Since $r^{\prime} \geqslant p^{\prime}$, we are done with replacement.

Separation is similar.

THEOREM 12. Let $\mathfrak{M} \vDash Z F C$ be countable, non-well-founded, and expandable to a model of $G B+\Delta_{1}^{1} C A$. Then $\mathfrak{M}$ is expandable to a model of $G B+\Sigma_{1}^{1} A C$.

Proof. We work in the countable, admissible set $\mathcal{H} \mathscr{\mathcal { Y }} \mathscr{P}_{\mathfrak{M}}$ (See Barwise [1975] for basic materials on $\mathcal{H} \mathscr{\mathcal { Y }} \mathcal{P}_{\mathfrak{M}}$. ) Let $\alpha\left(=\kappa^{\mathfrak{M}}\right)$ be the ordinal of $\mathcal{H} \mathcal{Y} \mathscr{P}_{\mathfrak{M}}$. Let $T$ be the theory $\mathrm{ZF}_{L(\alpha)}$ with replacement and separation axioms for formulas involving 
the extra symbol $\leqslant$, plus an axiom asserting that $<$ is a well-ordering of the model, plus an axiom asserting that the universe of the model is $\mathfrak{M}$ and that the $\in$ of the new model is the $\in$ of the original $\mathfrak{M}$. By Theorem 11, T is consistent. Clearly it is $\Sigma$ on $\mathscr{H} \mathscr{Y} \mathscr{P}_{\mathfrak{P}}$. Hence, by another theorem of Ressayre (or just the Gödel compactness theorem for $\alpha=\omega$ ), we can find such an $\langle\mathfrak{M}$, $<\rangle$ so that $\kappa^{\langle\mathfrak{R},<\rangle}=\alpha$. (The theorem of Ressayre is Proposition II.1 of Ressayre [1977]. It was rediscovered in Schlipf [1978, Theorem 3.3]. Similar results have also been proved by Friedman and Nadel.) But then let $\mathcal{X}=\mathscr{K} \mathcal{E}_{\langle\mathfrak{N},<\rangle}$. Then clearly $\langle\mathfrak{M}, \mathfrak{X}\rangle \vDash \mathrm{GB}$ $+\Sigma_{1}^{1} \mathrm{AC}$.

Corollary 13. $G B+\Sigma_{1}^{1} A C$ is a conservative extension of ZFC for sentences about sets.

A series of related conservative extension type results is poved in $\$ 2$ of Barwise and Schlipf [1976] for the theory Peano arithmetic. Analogous results hold here, for $\mathrm{ZF}, \mathrm{ZFC}$, or $\mathrm{ZF}+(V=O D)$, depending upon how much choice is used in the proof. Similar results for the definable subsets of a model are proved in Kreisel [1965]. These also can be generalized with our techniques to results about GB $+\Delta_{1}^{1}$ CA. In $\$ 3$ of the Barwise-Schlipf paper certain theorems about the hard core of a theory over a model are proved. Analogous results hold here. Finally, it is worth noting that the non-well-founded models of $\mathrm{ZF}$ actually expandable to models of $\mathrm{GB}+\Delta_{1}^{1} \mathrm{CA}$ are rather badly non-well-founded. For the non- $\omega$-standard expandable models, i.e., for the recursively saturated models, this is studied in $\$ 3$ of Schlipf [1978]. Many analogous results hold for $\alpha$-standard but non-well-founded models. It is fairly easy to prove, for example, that a model $\mathfrak{M}$ of $\mathrm{ZF}$ is recursively saturated iff it is non- $\omega$-standard and there are arbitrarily large ordinals (in the sense of $\mathfrak{M}$ ) $\alpha$ such that $R(\alpha)$ (in the sense of $\mathfrak{M}$ ) is an elementary submodel of $\mathfrak{M}$. (One direction of this is proved in that article; the other is easy.)

\section{REFERENCES}

J. Barwise [1975], Admissible sets and structures, Springer-Verlag, Berlin.

J. Barwise and J. Schlipf [1976], On recursively saturated models of Peano arithmetic, Model Theory and Algebra: A Memorial Tribute to Abraham Robinson (D. H. Saracino and B. Weispfenning, Eds.), Lecture Notes in Math., vol. 498, Springer-Verlag, Berlin, pp. 42-54.

K. Bielinski [1977], Extendability of structures as an infinitary property, Set Theory and Hierarchy Theory. V (A. Lachlan, M. Srebrny, and A. Zarach, Eds.), Lecture Notes in Math., vol. 619, Springer-Verlag, Berlin, pp. 75-94.

A. Ehrenfeucht and G. Kreisel [1966], Strong models of arithmetic, Bull. Acad. Polon. Sci. Sér. Sci. Math. Astronom. Phys. 14, pp. 107-110.

U. Felgner [1971], Comparison of the axioms of local and universal choice, Fund. Math. 71, pp. 43-62.

H. Gaifman [1975], Global and local choice functions, Israel J. Math. 22, pp. 257-265.

L. Harrington, L. Kirousis and J. Schlipf [1978], A generalized Kleene-Moschovakis theorem, Proc. Amer. Math. Soc. 68, pp. 209-213.

H. J. Keisler [1973], Forcing and the omitting types theorem, Studies in Model Theory (M. D. Morley, Ed.), Math. Assoc. of Amer., Buffalo, N. Y., pp. 96-133.

G. Kreisel [1965], Axioms for explicitly definable properties, Appendix A to Informal rigour and completeness proofs, Problems in the Philosophy of Mathematics (Imre Lakatos, Ed.), North-Holland, Amsterdam, pp. 162-165.

Y. N. Moschovakis [1971], Predicative classes, Axiomatic Set Theory, Part 1, Proc. Sympos. Pure Math., vol. 131, Amer. Math. Soc., Providence, R. I., pp. 247-264. 
[1974], Elementary induction on abstract structures, North-Holland, Amsterdam.

J. P. Ressayre [1977], Models with compactness properties relative to an admissible language, Ann. Math. Logic 11, pp. 31-55.

J. Schlipf [1977], A guide to the identification of admissible sets above structures, Ann. Math. Logic 12, pp. 151-192.

[1978], Toward model theory through recursive saturation, J. Symbolic Logic 43, pp. 183-206.

Department of Mathematics, California Institute of Technology, Pasadena, California 91109

Current address: Department of Mathematics, St. Mary's College of Maryland, St. Mary's City, Maryland 20686 